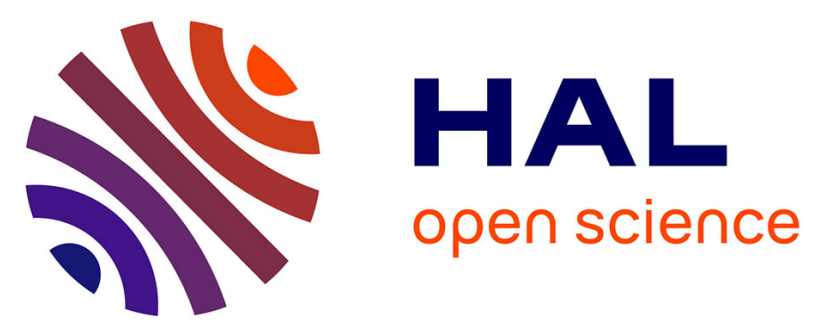

\title{
Total cross sections for ionizing processes induced by proton impact on molecules of biological interest: a Classical Trajectory Monte Carlo approach.
}

\author{
H. Lekadir, I. Abbas, C. Champion, J. Hanssen
}

\section{- To cite this version:}

H. Lekadir, I. Abbas, C. Champion, J. Hanssen. Total cross sections for ionizing processes induced by proton impact on molecules of biological interest: a Classical Trajectory Monte Carlo approach.. The Seventh International Symposium on Swift Heavy Ions in Matter, Jun 2008, Lyon, France. hal00252106

\section{HAL Id: hal-00252106 \\ https://hal.science/hal-00252106}

Submitted on 19 Jul 2008

HAL is a multi-disciplinary open access archive for the deposit and dissemination of scientific research documents, whether they are published or not. The documents may come from teaching and research institutions in France or abroad, or from public or private research centers.
L'archive ouverte pluridisciplinaire HAL, est destinée au dépôt et à la diffusion de documents scientifiques de niveau recherche, publiés ou non, émanant des établissements d'enseignement et de recherche français ou étrangers, des laboratoires publics ou privés. 
Total cross sections for ionizing processes induced by proton impact on molecules of biological interest: a Classical Trajectory Monte Carlo approach.

\author{
H. Lekadir, I. Abbas, C. Champion and J. Hanssen. \\ Université Paul Verlaine Metz, Laboratoire de Physique Moléculaire et des Collisions, \\ Institut J. Barriol FR CNRS 2843, 1 Bd Arago, 57078 Metz, Cedex3.
}

\begin{abstract}
In the current work, we present a study of ionizing interactions between protons and molecular targets of biological interest like water vapour and DNA bases. Total cross sections for single and multiple ionizing processes are calculated in the Independent Electron Model and compared to existing theoretical and experimental results for impact energies ranging from 10keV/amu to $10 \mathrm{MeV} / \mathrm{amu}$. The theoretical approach combines some characteristics of the Classical Trajectory Monte Carlo method with the Classical Over-Barrier framework. In this "mixed" approach, all the particles are described in a classical way by assuming that the target electrons are involved in the collision only when their binding energy is greater than the maximum of the potential energy of the system \{projectile-target\}. We test our theoretical approach on the water molecule and the obtained results are compared to a large set of data and a reasonable agreement is generally observed specially for impact energies greater than $100 \mathrm{keV}$, excepted for the double ionization process for which large discrepancies are reported. Considering the DNA bases, the obtained results are given without any comparison since the literature is till now very poor in terms of cross section measurements.
\end{abstract}

PACS: $34.70 .+e$, 82.30. Fi

Keywords: charge exchange, ionization, biological molecules

Corresponding author: Tel: +33 (0)3-87-31-58-98 , Fax: +33 (0)3-87-54-72-57

E-Mail: jocelyn@univ-metz.fr (J. Hanssen). 


\section{Introduction}

Understanding and describing at the finest scale the ionizing processes induced by charged particles on biomolecular targets like water vapour and DNA nucleobases (Adenine, Cytosine, Guanine and Thymine) are of prime importance in many fields including radiobiology, radiotherapy and medical imaging [1-3]. Consequently, it remains today crucial to access to differential and total cross sections to develop accurate numerical simulations of charged particle transport in biological matter. We investigate in this work the ionizing interactions induced by proton impact on water molecule and DNA nucleobases. Total cross sections are then determined in the Independent Electron Model (IEM) for single and multiple processes, namely for single capture $S C$, single ionization $S I$, double capture $D C$, double ionization $D I$ and capture+ionization $C I$.

On the experimental side, single processes induced by protons on water molecule have been extensively studied. We can at first mention the work of Dagnac et al. [4] where total cross section measurements have been reported for $S I$ and $S C$ induced by protons with impact energies ranging from 2 to $60 \mathrm{keV}$. Later on, Rudd et al. [5] have published SI and SC total cross sections for 7-4000keV protons whereas Bolorizadeh and Rudd [6] reported doubly differential (differential in energy and angular transfers) and total SI cross sections for proton energies ranging from 15 to $150 \mathrm{keV}$. More recently, Toburen [7] have reported total cross sections for electron capture by protons on water vapour. Considering the multiple processes, we only find in the literature measurements for heavier projectiles like $\alpha$-particles [8] and $\mathrm{Xe}^{44+}$ ions [9]. On the theoretical side, the single processes are well-documented whereas the multiple processes have been less studied. For SI process, we can then cite the work of Long et al. [10] based on the density-functional theory, which provides doubly, singly differential and total cross sections. More recently, a very good agreement with experimental doubly differential and total cross section measurements has been reported for protons in water by Olivera et al. [11] in the continuum distorted wave-eikonal initial state (CDW-EIS) framework 
- for ionization as well as for electron capture - and by Boudrioua et al. [12] in the first Born approximation for ionization. Considering the double ionization of water, we only find in the literature the theoretical work of Gervais et al. [14] performed in the CDW-EIS approximation in which the target electrons are treated as independent particles (IEM model). Very recently, Errea et al. [13] have reported total cross sections for $S I$ and $S C$ in the eikonal-classical trajectory Monte Carlo (CTMC) framework. Moreover, these authors have also provided total cross sections for the $C I$ process.

Considering now the nucleobase ionization by heavy charged particles, the literature is very poor. Nevertheless, we can cite the study of De Vries et al. [15] about Uracil excitation and fragmentation by $C^{q+}(q=1-6)$ ions and more recently the work of Moretto-Capelle et al. [16] dedicated to electron spectroscopy of dry gas-phase Uracil base by proton ions.

In the present work, we briefly present the theoretical model used to calculate the total cross sections for all the single and multiple processes induced by proton impact on water and nucleobases. Our results are reported and compared to available experimental, semi-empirical and theoretical results.

Finally, note that atomic units $\left(e=m_{0}=r_{0}=\hbar=1\right)$ are used hereafter unless noted otherwise.

\section{Theory}

In this paper, we use the CTMC model developed by Abbas et al. [17] and recently modified to treat the multiple ionizing processes in water. In fact, in its first version, the code described the multiple processes in a correlation-multi-electronic approach whereas in its present form, the multiple processes are treated in the Independent Electron Model, i.e. in a more convenient way for studying large molecular targets like those involved in the present work. Compared to the "standard" CTMC simulations which are, for the major part, based on 
boundary conditions taken from a quantum description of the target electronic distribution [13], our model uses the Classical Over-Barrier (COB) criteria [18-19].

In this approach, all the particles are described via classical Newtonian laws and a given target electron is ejected only when its energy becomes greater than the sum of all the potential energies induced by the other existing particles. The simulation starts and stops at a sufficiently large time $t$, i.e. for a large inter-nuclear distance $R \cong 100$ a.u., these latter being linked via the equation $\vec{R}=\vec{b}+\vec{v} t$. For each simulation, the initial conditions are defined by the projectile velocity $\vec{v}$ and the impact parameter $\vec{b}$ and we determine, for each time step $\Delta t\left(\cong 10^{-2} a . u\right.$. $)$, a new position and a new velocity for the projectile and for the secondary electron potentially ejected. Thus, by taking the initial position of the target as fixed in the laboratory frame and by considering that the potential seen by the ejected electron of the target is given by

$$
V(\vec{r})=-\frac{Z_{P}}{|\vec{r}-\vec{R}|+a}-\frac{Z_{T}}{\vec{r}+a}
$$

we consider that the electron ejection needs two prerequisites:

i) at each time step $\Delta t$, the maximum potential energy denoted $\operatorname{Max}[V(\vec{r})]=V_{\operatorname{Max}}$ has to be lower than the binding target energy $E_{b}\left(E_{b}<0\right)$,

ii) the ratio $\Delta t / t_{e}$, where $t_{e}$ denotes the classical orbital period of the target electron given by $t_{e}=2 \pi Z_{T} \frac{1}{\sqrt{2\left|E_{b}\right|^{3}}}\left(\right.$ with $\Delta t<t_{e}$ ), has to be greater than a random number $\delta$ (chosen between 0 and 1 ); this condition has been introduced to compensate the lack, in this model, of target electron spatial density representation.

Note that in Eq.(1) $Z_{P}$ and $Z_{T}$ represent the projectile and the target charge, respectively: here, $Z_{T}$ is taken equal to 1 . The vector $\vec{r}$ denotes the electron position whereas the parameter $a$ is seen as an adjustable numerical cut-off (Abbas et al. [17]) whose stability region was found around $a \cong 0.05 a . u$. 
Thus, if the conditions $i$ ) and ii) are satisfied, an electron is ejected with a kinetic energy $T_{e}=\frac{1}{2} v_{e}{ }^{2}=E_{b}+\frac{Z_{T}}{r+a}$ and emitted in a random direction from a position randomly selected within a sphere centered on the target.

At the end of each simulation (i.e. at large $R$ ), the electron-projectile energy $H_{P}$ and the electron-target energy $H_{T}$ are evaluated and given by

$$
H_{P, T} \approx \frac{1}{2}\left(\vec{v}_{e}-\vec{v}_{P, T}\right)^{2}-\frac{Z_{P, T}}{\left|\vec{r}-\vec{R}_{P, T}\right|},
$$

where $\vec{v}_{e}$ is the electron velocity and $\vec{v}_{P, T}$ and $\vec{R}_{P, T}$ the velocity and the position vector of the projectile $P$ and the target $T$ at large $R$, respectively. Thus, we have a capture (resp. a recapture) if $H_{P} \leq 0$ (resp. $H_{T} \leq 0$ ) or an ionization if $H_{P}>0$ and $H_{T}>0$.

Then, for a given projectile velocity $v$, the total cross section for a given single ionizing process $j(j=S C$ or $S I)$ is

$$
\sigma_{j}(v)=\sum_{i=1}^{I} \sigma_{j}^{i}(v)=\sum_{i=1}^{I} 2 \pi \int_{0}^{b_{\max }} b \cdot d b \cdot P_{j}^{i}(b),
$$

where the summation includes all the molecular subshell contributions ( $I=5$ for the water molecule and $\geq 20$ for the DNA bases). The upper limit of the integral, denoted $b_{\max }$, has been found of the order of $15 a . u$. for the systems studied here.

Note that the mono-electronic probability introduced in Eq.(3) is given by

$$
P_{j}^{i}(b)=\frac{N_{j}^{i}}{N},
$$

where $N_{j}^{i}$ is the number of simulations for which the process $j$ occurs among $N(\cong 500)$ simulations. Similar procedures are then repeated for a large number $(\geq 100)$ of impact parameters.

Finally, it seems us important to remind that the collisions considered here are many electron situations which have to be solved by introducing all the electronic correlations. To overcome this complexity, we used the independent electron model (IEM) approximation. Thus, inspired 
by the recent work of Errea et al. (2007) [13], we defined the mono-electronic probability $P_{e l}^{i}(b)$ for an electron of a molecular state $i$ to be not created as

$$
P_{e l}^{i}(b)=1-P_{S C}^{i}(b)-P_{S I}^{i}(b)
$$

Consequently, the exclusive double ionization DI probability is simply given by

$$
P_{D I}(b)=\sum_{i=1}^{I}\left(P_{S I}^{i}(b)\right)^{2} \prod_{l \neq i}\left(P_{e l}^{l}(b)\right)^{2}+2 \sum_{i, j=1}^{I} P_{S I}^{i}(b) P_{S I}^{j}(b) P_{e l}^{i}(b) P_{e l}^{j}(b) \underset{l \neq(i, j)}{\prod_{e l}}\left(P_{l}^{l}(b)\right)^{2}
$$

whereas the exclusive capture+ionization $C I$ probability is given by

$$
P_{C I}(b)=2 \sum_{i=1}^{I} P_{S C}^{i}(b) P_{S I}^{i}(b) \prod_{l \neq i}\left(P_{e l}^{l}(b)\right)^{2}+4 \sum_{i, j=1}^{I} P_{S C}^{i}(b) P_{S I}^{j}(b) P_{e l}^{i}(b) P_{e l}^{j}(b) \underset{l \neq(i, j)}{\prod_{e l}}\left(P_{l}^{l}(b)\right)^{2} .
$$

Finally, the total cross section for a double process $k=D I$ or $C I$ is given by

$$
\sigma_{k}(v)=2 \pi \int_{0}^{b_{\max }} b P_{k}(b) d b .
$$

\section{Results and discussion}

Contrary to the existing quantum mechanics models, which rapidly become untreatable for large molecules, our model is relatively simple and avoids needing any information about the quantum structure of the target except the knowledge of the binding energies. The scope of the current work is to carry out the calculation of the multiple ionizing processes induced by charged particles impinging molecular targets of biological interest. In a first part we are interested by the water molecule which represents the "simplest" molecule to model the biological matter whereas in a second part more complex molecules are studied, namely the four nucleobases of the DNA macromolecule. 


\subsection{Ionizing processes induced by protons on water vapour}

Figure 1 depicts a comparison between the obtained theoretical results to a large set of available experimental measurements and/or existing theoretical predictions in terms of total cross sections for $i$ ) the single ionization process (Panel a), $i$ ) the single capture process (Panel b) and iii) the multiple processes (Panel c).

In Panels a) and b), we observe that our results are in good agreement with all the data (experimental as well as theoretical) provided that the incident energies are greater than $100 \mathrm{keV}$ whereas for lower energies, large disagreement may be observed. In fact, the classical description of the collisions becomes obviously invalid in this low-energy regime and more sophisticated models - such as the CDW-EIS model - are needed (see the results reported by a dash-dotted line [14] in Panels a) and b)). In panel c), we report the calculated total cross sections for the multiple processes induced by protons, namely the capture+ionization $(C I)$ and the double ionization $(D I)$. Unfortunately, available data are rare in the literature and we only found theoretical predictions for describing the $C I$ [13] and $D I$ [14] processes. Whereas our theoretical results exhibit a very good agreement with the recent calculations of Errea et al. for describing the $C I$ process [13], we clearly observe an underestimation of the results reported by Gervais et al. in [14] (see Panel c)) for the DI process. This large disagreement is actually not well understood but highlights the limitation of the CTMC approach to treat the DI process as already mentioned by Reinhold and Burgdörfer [26] who have shown that in the IEM approximation, the double ionization was badly described by classical models. Finally, note that some of the SI and DI results reported in Panels a) and c) have been calculated for liquid water [14] whereas our results concern water vapor. However, we have recently performed SI and $D I$ cross section calculations by replacing in our code the binding energies of the water molecule by those corresponding to the liquid phase and only minor discrepancies were found $(\leq 6 \%)$ 


\subsection{Ionizing processes induced by protons on DNA bases}

To characterize the nucleobases targets, we have used the binding energies provided by M. F. Ruiz López (private communication) who used the quantum chemical GAUSSIAN code method which represents the highest performed code giving access to accurate energies for dynamical calculations. To our knowledge, no study has been reported for the processes under investigation in the present work and comparison to existing data remains still today impossible. However, the following remarks can be drawn:

- The overall behaviors of the total cross sections are similar to those reported for the water target.

- The magnitude of the cross sections seems to depend on the number of electronic states included in the target description and then proportional to the number of target electrons.

\section{Conclusion}

We have applied for high impact-energy protons a relatively simple classical model which combines some elements of the CTMC method and the $C O B$ approach to estimate the total cross sections for single and multiple ionizing processes induced on water target and nucleobases. The theoretical results also obtained exhibit a relatively good agreement with the existing experimental data (for water target) and permit to predict the behaviour of the multiple cross sections for ionizing processes occurring with other very important targets of biological interest such as the nucleobases. Thus, with regard to the great importance of this kind of studies in radiobiology, where the description of the primary interaction between ionizing particles and biomolecules like nucleobases is essential, we really hope that experimental data on these biological systems will be soon available. 


\section{Acknowledgements}

We would like to thank Pr. M. F. Ruiz López of the Nancy-University for its kind help. We also acknowledge the financial support provided by the 'Jean Barriol' Institute.

\section{References}

[1] H. A. Bethe, Ann. Phys. 5 (1930) 325.

[2] U. Fano, Ann. Rev. Nucl. Sci. 13 (1963) 1.

[3] M. Inokuti, Rev. Mod. Phys. 43 (1971) 297.

[4] R. Dagnac, D. Blanc and D. Molina, J. Phys. B: At. Mol. Opt. Phys. 3 (1970) 1239.

[5] M. E. Rudd, T. V. Goffe, R. D. DuBois and Toburen L H, Phys. Rev. A 31 (1985) 492.

[6] M. A. Bolorizadeh and M. E. Rudd, Phys. Rev. A 33 (1986) 888.

[7] L. H. Toburen, Radiat. Env. Bio. 37 (1998) 221.

[8] M. E. Rudd, A. Itoh and T. V. Goffe, Phys. Rev. A 32 (1985) 2499.

[9] G. H. Olivera, P. D. Fainstein, and R. D. Rivarola, Phys. Med. Biol. 43 (1998) 2347.

[10] K. A. Long and H. G. Paretzke, J. Chem. Phys. 95 (1991) 1049.

[11] G. H. Olivera, P. D. Fainstein and R. D. Rivarola, Phys. Med. Biol. 41 (1996) 1633.

[12] O. Boudrioua, C. Champion, C. Dal Cappello and Y. V. Popov, Phys. Rev. A 75 (2007) 22720.

[13] L. F. Errea, C. Illescas, L. Mendez, B. Pons, I. Rabadan, and A Riera., Phys. Rev A 76 (2007) 040401(R).

[14] B. Gervais, M. Beuve, G. H. Olivera and G. E. Galassi , Radiat. Phys. Chem. 75 (2006) 493.

[15] J. De Vries, R. Hoekstra, R. Morgenstern and T. J. Schlathölter, J. Phys. B: At. Mol. Opt. Phys. 35 (2002) 4373.

[16] P. Moretto-Capelle and A. Le Padellec, Phys. Rev. A 74 (2006) 062705. 
[17] I. Abbas, C. Champion, B. Zarour, B. Lasri and J. Hanssen, Phys. Med. Biol. 53 (2008) N41-N51.

[18] A. Bárány et al., Nucl. Instr. and Meth. B 9 (1985) 397

[19] A. Niehaus, J. Phys. B: At. Mol. Opt. Phys. 19 (1986) 2925

[20] R. Abrines and I. C. Percival, Proc. Phys. Soc. 88 (1966) 861

[21] W. E. Wilson, J. H. Miller and L. H. Toburen, J. Chem. Phys. 80 (1984) 5631.

[22] H. Date, K. L. Sutherland, T. Hayashi, Y. Matsuzaki and Y. Kiyanagi, Radiat. Phys. Chem. 75 (2006) 179.

[23] S. Endo, E. Yoshida, H. Nikjoo, S. Uehara, M. Hoshi, M. Ishikawa and K. Shizuma, Nucl. Instr. and Meth. B 194 (2002) 123.

[24] P. D. Fainstein, G. H. Olivera and R. D. Rivarola, Nucl. Instr. and Meth. B 107 (1996) 19.

[25] F. Gobet, B. Farizon, M. Farizon, J. M. Gaillard, M. Carré, M. Lezius, P. Scheier and T. D. Mark, Phys. Rev. Lett. 86 (2001) 3751.

[26] C.O. Reinhold and J. Burgdörfer J. Phys. B: At. Mol. Opt. Phys. 26 (1993) 3103

\section{Figure captions}

Figure 1:

Total cross sections for single and multiple ionizing processes induced by protons on water target. Comparison between our theoretical results (solid line) and available data. Panel a): Ionization process: experimental measurements taken from Rudd et al. [5] (stars), Wilson et al. [21] (solid squares), Date et al. [22] (solid up triangles), and theoretical predictions taken from Gervais et al. [14] (dash-dotted line), Boudrioua et al. [12] (shortdash-dotted line), Endo et al. [21] (dotted line), Errea et al. [13] (dashed line) and Fainstein et al. [24] (dash-dot-dotted line). Panel b): Capture process: experimental measurements taken from Dagnac et al. [4] (solid down triangles), Gobet et al. [25] (solid squares), Toburen [7] (solid circles), Date et al. [22] (solid up triangles) and theoretical results taken from Errea 
et al. [13] (dashed line), Endo et al. [23] (dotted line) and Fainstein et al. [24] (dash-dotdotted line). Panel c): Multiple processes: theoretical predictions taken from Errea et al. [13] (dashed line) for the $C I$ process and Gervais et al. [14] for the DI process (stars and dotted line).

Figure 2:

Total cross sections for single and multiple ionizing processes induced by protons on DNA bases.

\section{Figure 1}

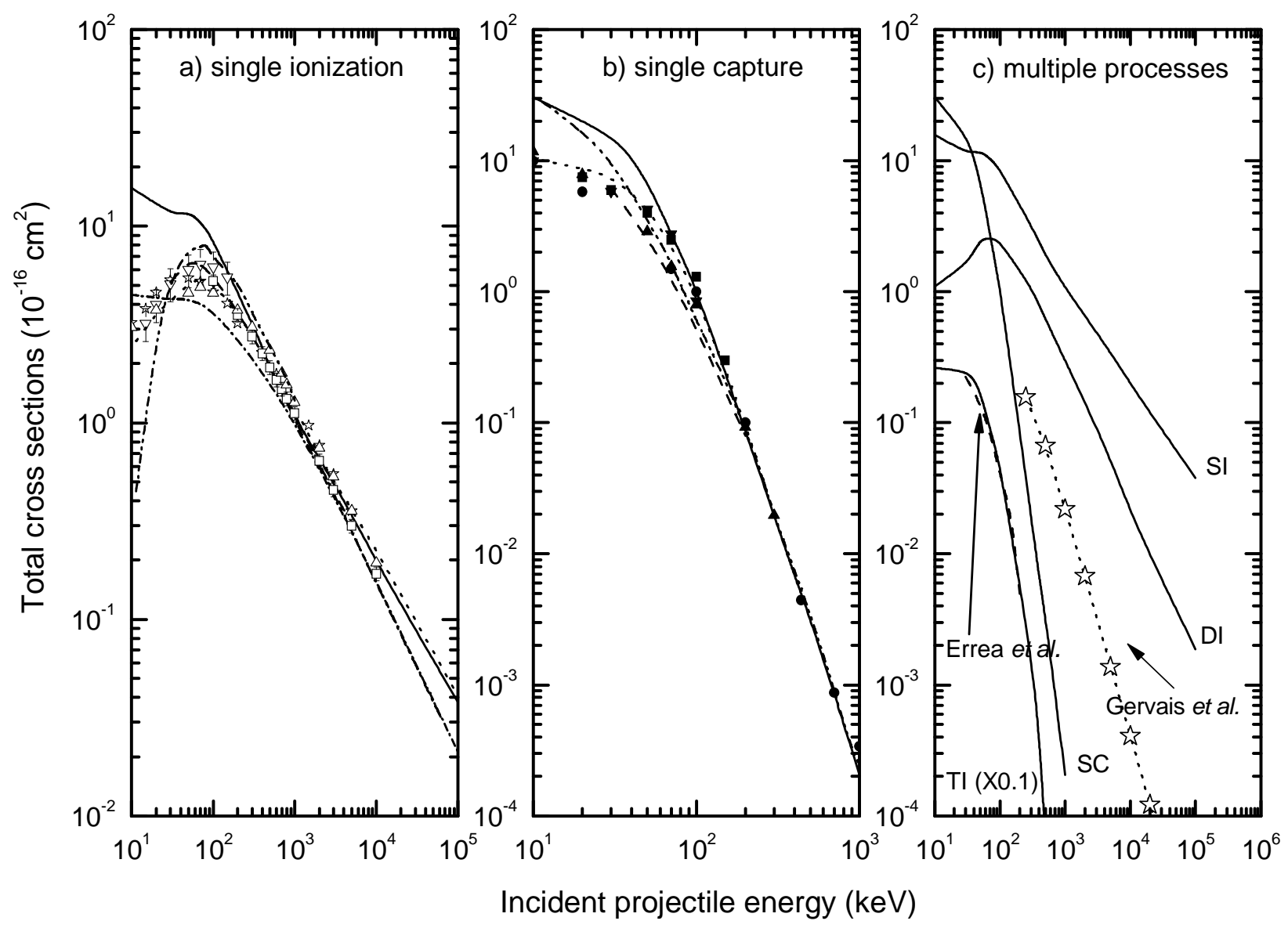


Figure 2

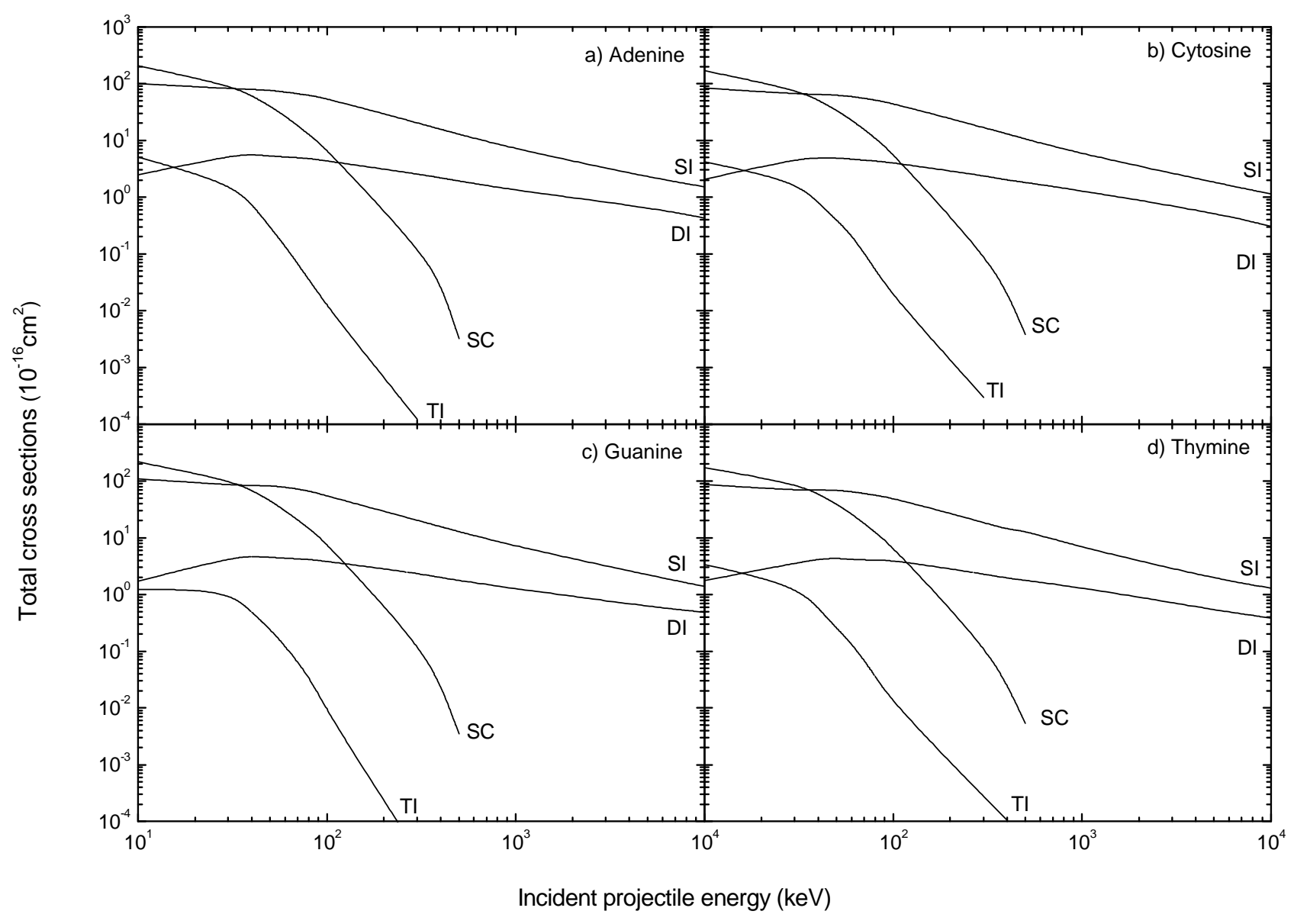

